\title{
Daten zur Multiplen Sklerose in Deutschland und ihre Abbildung im Register des ambulanten Netzwerkes NeuroTransData (NTD)
}

\section{Data on multiple sclerosis in Germany and their representation in the ambulatory registry NeuroTransData (NTD) network}

\author{
Autoren \\ Institute \\ 1 Neuro Trans Data \\ Schlüsselwörter \\ Key words \\ Multiple sclerosis, Epidemiology, Registry \\ eingereicht 15.05 .2019 \\ akzeptiert $\quad 28.02 .2020$ \\ Bibliografie \\ DOI https://doi.org/10.1055/a-1130-6222 \\ Fortschr Neurol Psychiatr 2020; 88: 379-385 \\ (c) Georg Thieme Verlag KG Stuttgart · New York \\ ISSN 0720-4299 \\ Korrespondenzadresse \\ Prof. Markus Weih \\ Facharzt für Neurologie, Medic Center Nürnberg \\ Schweinauer Hauptstr. 43 \\ 90441 Nürnberg \\ Germany \\ Tel.: $0911 / 667085$ \\ Fax: $0911 / 620503$ \\ E-Mail: m.weih@mediccenter.net
}

Markus Weih', Fabian Roßnagel', Heidi Dikow' ${ }^{1}$, Knut Wehrle', Stefan Braune', Arnfin Bergmann'

Multiple Sklerose, Registerdatenbank, Versorgungsforschung

\section{ZUSAMMENFASSUNG}

Anliegen und Methodik Beschreibung der Basisdaten, der häufigsten Symptome und deren medikamentöser, nichtmedikamentöser und kombinierter symptomatischer Behandlung in einer großen Stichprobe von MS-Patienten, die sich in ambulanter Behandlung der Mitgliedspraxen des NeuroTransData (NTD)-Arztnetzwerkes in Behandlung befinden.

Ergebnisse Aktuell sind 21407 Patienten im Register. Das Durchschnittsalter betrug 49,0 \pm 13,0 Jahre, der Frauenanteil lag bei 72,3\%, die Krankheitsdauer bei 14,3 $\pm 8,9$ Jahren. Eine schubförmig-remittierende MS (RRMS) lag in $77 \%$ vor, $15 \%$ sekundär-progrediente MS, primär-progrediente MS (PPMS) 5\%. Der durchschnittliche EDSS-Score der
Gesamtstichprobe lag bei 2,8 (Range 0,5-8). Fatigue war in allen Subtypen das häufigste Symptom (96\%), gefolgt von der Spastik (31\%), v.a. bei der SPMS (47\%) und PPMS (36\%). Hinsichtlich der medikamentösen, nichtmedikamentösen und kombinierten symptomatischen Behandlung zeigte sich eine große Variationsbreite. Während in der Gesamtpopulation die Spastik in $81 \%$ behandelt wurde, erhalten nur $21 \%$ der Patienten eine Behandlung der Fatigue. Auch Defäkations- und Miktionsstörungen waren in unserer Stichprobe häufig noch unbehandelt (69\% bzw. 56\%).

Schlussfolgerungen Aufbau, kontinuierliche Weiterentwicklung und Pflege eines Registers stellen bei komplexen und chronischen Erkrankungen wie der MS ein wichtiges Instrument zur Beurteilung und damit auch zur Verbesserung und zum Vergleich der Behandlungsart und ihrer Defizite dar.

Unsere Ergebnisse sind methodisch schwer vergleichbar mit der Stichprobe des MS-Registers der DMSG. Die Fatigue war aber in beiden Registern das häufigste Symptom.

\section{ABSTRACT}

Aims and methodology Description of basic data, common symptoms and their medical, non-drug and combined symptomatic treatment in a large sample of MS patients undergoing outpatient treatment of the German NeuroTransData (NTD) physician network.

Results Currently there are 21,407 patients in the registry. Average age is $49.0 \pm 13.0$ years, $72,3 \%$ of them female, average disease duration is $14.3 \pm 8,9$ years. Relapsingremitting MS (RRMS) was present in $77 \%$, secondary-progressive MS (SPMS) 15\%, PPMS 5\%. The mean EDSS score of the total sample was 2.8 (range 0,5-8). Fatigue was the most common symptom in all subtypes (96\%), followed by spasticity (all 31\%, SPMS: 47\%; PPMS 36\%). Regarding symptomatic drug treatment, non-drug treatment and combined treatment, there was a wide range of variation. While spasticity was treated in $81 \%$, only $21 \%$ of patients with fatigue receiving any form of therapy, Also, fecal and urine incontinence often remained untreated in $69 \%$ resp. $56 \%$ of cases. 
Conclusions Setup, development and maintenance of a registry for a complex and chronic disease like MS represents an instrument to assess and improve patient care in the outpatient setting. Our results are hard to compare with the
DMSG-registry, another German, more hospital-based data collection. However, both registries identify fatigue as the most common symptom in MS.

\section{Einleitung}

Die Multiple Sklerose (MS) ist die häufigste chronisch-entzündliche Erkrankung des zentralen Nervensystems (ZNS) bei jungen Erwachsenen. Nach aktuellen europäischen Leitlinien beträgt die Prävalenz in Europa ca. 700000 und weltweit ca. 2,5 Millionen Fälle. Die Inzidenz variiert nach Breitengrad, üblicherweise treten 8-10 Neuerkrankungen pro 100000 auf [1].

Die genaue Ursache der Erkrankung ist nicht bekannt, es besteht aber weitgehender wissenschaftlicher Konsens, dass die Erkrankung multifaktoriell bedingt ist. Bei den von der MS histopathologisch, pathogenetisch und therapeutisch als eigenständige Entität abgrenzbaren Neuromyelitis-optika Spektrumserkrankungen (NMOSD) sind hingegen oft Aquaporin-4-Antikörper nachweisbar.

Die MS gilt als nicht heilbar, inzwischen aber als gut behandelbar und verläuft in der Mehrzahl der Fälle schubförmig-remittierend (relapsing remitting multiple sclerosis, RRMS). Weitere Verlaufsformen sind das klinisch isolierte Syndrom (KIS oder CIS), die sekundär progrediente MS (secondary progressive multiple sclerosis, SPMS) und die primär progrediente MS (primary progressive multiple sclerosis, PPMS).

In vielen Fällen führt die Erkrankung trotz therapeutischer Fortschritte zu fortschreitender und bleibender Behinderung, sodass etwa ein Drittel bis die Hälfte der Patienten trotz Behandlung erhebliche Funktionseinschränkungen entwickeln und oft berufsunfähig werden. Die Erkrankung ist mit hohen und steigenden Kosten verbunden. Hier ist seit den 1990er-Jahren von einem Kostenanstieg von ca. $10000 €$ auf aktuell bis $63000 € /$ Jahr auszugehen. Die Kosten für einen Schub wurden auf $2500 €$ geschätzt [2].

Aufgrund neuerer Daten ist davon auszugehen, dass sowohl die Inzidenz als auch die Prävalenz der MS in den westlichen Industrieländern hoch ist und weiter zunimmt. Die MS zeigt eine geografische Verteilung, ist im Bereich des Äquators selten und steigt im nördlichen und südlichen Breitengrad wieder an. Aktuell wird in Mitteleuropa von einer Prävalenz von 8-10/100 000 und einer Inzidenz von 3-6/ 100000 ausgegangen. Nach Resultaten einer dänischen Langzeitstudie über 60 Jahre ist die aktuelle Inzidenz vielleicht sogar noch höher. Auch andere Datenquellen vermuten eine Zunahme der Inzidenz, wie eine andere große Stichprobe aus Kanada vermuten lässt [3]. Auch nach einer aktuellen systematischen Analyse der Global Burden of Disease Study stieg weltweit die Prävalenz der MS zwischen 1990 und 2016 um 10,4\% auf über 2 Millionen Fälle an [4]. In Deutschland wurde lange von einer Prävalenz von etwa 100000 bis maximal 130000 Erkrankten ausgegangen. Erst mit Analyse neuerer Daten aus der kassenärztlichen Versorgung bestätigte sich in den letzten Jahren die Vermutung, dass in Wirklichkeit mehr Patienten betroffen sind [5]. So wurde zuletzt 2017 in einer populationsbasierten Studie des Zentralinstituts für die kassenärztliche Versorgung in Deutschland ein Anstieg von etwa 172000
Patienten im Jahr 2009 auf über 223000 MS-Erkrankte 2015 beschrieben [6].

Die Ursachen dieses vermutlich weltweiten Anstiegs der Prävalenz, ggf. auch der Inzidenz, sind noch unklar. Vermutet werden geänderte diagnostische Kriterien, bessere Zugänglichkeit von bildgebenden Verfahren, verbesserte kausale und symptomatische, medizinische und nichtmedikamentöse Therapiemöglichkeiten, bessere Palliativ- und Rehabilitationsmedizin, aber auch allgemein eine Zunahme von Autoimmunerkrankungen, ggf. im Rahmen einer komplexen Interaktion des Immunsystems mit genetischen Faktoren und Umweltfaktoren.

Zwar bestehen bereits Maßnahmen zur Verbesserung der ambulanten Versorgung von MS-Patienten, diese fokussierten in der Vergangenheit aber oft auf den stationären Bereich [7]. Im ambulanten Bereich greift seit 2012 das GKV-Versorgungsstärkungsgesetz (GKV-VSG), welches explizit bei schweren Verlaufsformen der MS eine ambulante spezialfachärztliche Versorgung erlaubt [8].

2016 hat das Institut für Gesundheits- und Sozialforschung (IGES) festgehalten, dass die Behandlungsplanung nicht ausreichend an die Krankheitsaktivität angepasst sei und die symptomatische Therapie große Defizite aufweise; es forderte folgerichtig strukturierte, besser vernetzte Versorgungskonzepte [9].

Basis jeder strukturierten Datenerhebung und Versorgung sollten wissenschaftliche Register sein. Jedoch waren in Deutschland mit seinem breitgefächerten Gesundheitswesen entsprechende Studien schwer durchführbar. Im Gegensatz dazu gibt es in manchen skandinavischen Ländern durch die abweichende Organisation der Sozialversicherungen bzw. des Gesundheitswesens bereits seit Jahrzehnten epidemiologische Studien zur MS. Beispielhaft sei das schon 1948 gegründete dänische Register genannt [10]. In den letzten Jahren konnten jedoch in Deutschland und europaweit Fortschritte erzielt werden. So hat der Bundesverband der Deutschen Multiple Sklerose Gesellschaft (DMSG) bereits 2001 ein Register initiiert. 2008 berichtete Flachenecker im Deutschen Ärzteblatt erstmals über die Versorgungssituation der MS-Patienten in Deutschland [11]. Bis 2018 hatte sich das Register der DMSG bis auf über 35000 Patienten ausgebaut [12].

2015 wurde von der EMA eine „Patient Registries Initiative“ gestartet [13]. 2017 erfolgte hier ein erster Workshop, der unter anderem standardisierte Kerndatensätze, gemeinsame Terminologien und Definitionen forderte [14]. Gegenwärtig von Bedeutung sind die European Multiple Sclerosis Platform (EMSP) und die Big MS Data Group. International ist MSBase zu nennen, mit aktuell fast 70000 Patienten aus 35 Ländern [15]. In der vorliegenden Arbeit sollen die Entwicklung, Methodik, die krankheitsbezogenen Grunddaten und die Ergebnisse zu Häufigkeit der 
medikamentösen und nichtmedikamentösen symptomatischen Behandlung von MS-Patienten im Rahmen des bislang weniger bekannte Register des NeuroTransData Ärztenetzwerkes vorgestellt werden.

\section{Methoden und Beschreibung des Registers}

Das Register wurde 2003 von 12 niedergelassenen Neurologen gegründet. Aktuell tragen 133 Neurologen aus 66 Praxen Daten bei [16]. Die Praxen versorgen etwa 600000 ambulante Patienten pro Jahr. Seit 2013 werden die Daten in einer webbasierten Plattform erfasst, sind verschlüsselt und pseudonymisiert. Das Register wurde 2012 von der Ethik-Kommission der Bayerischen Ärztekammer positiv votiert (Nr. 11144; Antrag vom 18.5.2012; Schreiben vom 14.6.2012) und am 25.4.2017 auch von der LÄK Nordrhein.

Die Daten sind konform mit nationalen, europäischen und internationalen Standards (BDSG, EU-DSGVO, Guidelines for Good Pharmacoepidemiology Practices [GPP] der International Society for Pharmacoepidemiology [ISPE] 2016, Strengthening the reporting of observational studies in Epidemiology [STROBE], EMA requirements for the use of patient registries for regulatory purposes -methodological and operational considerations; 5.11.2018, EMA / 763513/2018 und der Deklaration von Helsinki).

Die Aktivitäten des Registers werden ermöglicht, indem es für die Datendokumentation, Teilnahme an Forschungsprojekten und Studien eine vertraglich transparent festgelegte finanzielle Aufwandsentschädigung gibt, um die dafür notwendigen personellen und infrastrukturellen Ressourcen in den Praxen unterhalten und weiterentwickeln zu können. Die NTD GmbH trägt den Aufwand der Infrastruktur des Registers durch Erlöse aus der Tätigkeit als CRO [17] und als Studienzentrum und erhält hierüber mit der pharmazeutischen Industrie vertraglich genau festgelegte und transparente Einkünfte.
Auf der Grundlage einer geschätzten Zahl von 220000 MSPatienten entspricht der Umfang des NTD-MS-Registers ca. 9,7\% aller MS-Patienten in Deutschland.

Alle Daten des Registers wurden selbst erhoben bzw. stammen nicht aus anderen, externen Datenquellen. Alle MS-Patienten werden standardisiert neurologisch in den Praxen untersucht. Der Funktionsstatus wird standardisiert unter Verwendung des EDSS ermittelt. In NTD ist es gewünscht, dass die Ärzte als EDSS-Rater über www.neurostatus.org zertifiziert sind, dies stellt jedoch keine Grundvoraussetzung gemäß Satzung dar. Die Qualitätsstandards für die teilnehmenden Praxen umfassen (neben dem Facharztstandard) u.a.: Zertifizierung der Praxis nach DINEN ISO 9001, Weiterbildungsbefugnis im Fachgebiet vorhanden oder innerhalb eines Jahres realisierbar, kontinuierliche Fortbildung für Ärzte und MFA (Webinar und Präsenzveranstaltungen). Zum Eintritt in das Register wird dem Patienten eine Patienteninformation und Einverständniserklärung gegeben. Die Patientenunterschriften werden in den Praxen aufbewahrt.

Die Dateneingabe erfolgt entweder manuell oder direkt von digitalen Quellen unter Monitoring des DatenmanagementTeams. Anschließend erfolgt eine automatische fachlich-inhaltliche Überprüfung auf Vollständigkeit, Inkonsistenzen und Korrektheit mit automatischer oder manueller Rückmeldung von 55 Items mindestens einmal/Woche (Querie). Jeder Eintrag in die Datenbank wird protokolliert (log).

Die in >Abb. 1 aufgeführten Symptome (inkl. Tremor, Ataxie) wurden durch die study nurses aus der klinischen Routinedokumentation (neurologischer Befund oder Diagnose) des Arztes entnommen. Die Fatigue wurde über den validierten FSMC-Fragebogen (Fatigue Skala für Motorik und Kognition) erhoben [18]. Kognitive Störungen werden über den Demtect oder Mini-Mental Status Test (MMST) erhoben. Es werden alle Einzelitems gespeichert. Als Cutoff für eine kognitive Störung wurden Demtect-Werte unter 13 von 18 bzw. unter 27 von 30 (MMST) verwendet. Ob die Stichprobe repräsentativ ist, wurde nicht gesondert ermittelt.

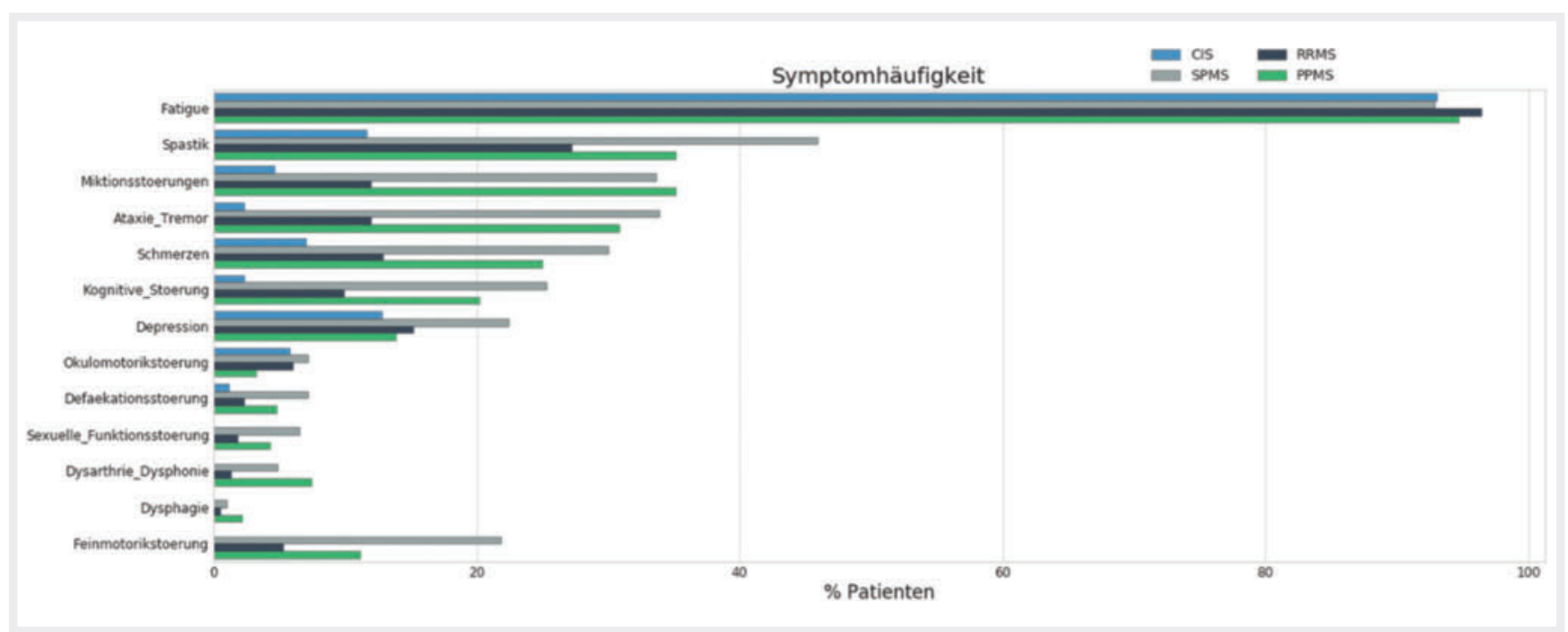

-Abb. 1 Symptomhäufigkeit bei MS. 
Für die vorliegende Analyse erfolgte ein Export strukturierter Daten zwischen dem 1.1.2010 und dem 31.12.2018 mit Fokus auf symptomatische Therapie. Hinsichtlich medikamentöser Behandlung wurden die Daten zu Wirkstoffen (Baclofen, Antidepressiva usw.) und Zeitdauern der Rezeptierungen aus der Praxissoftware bzw. Routineversorgung manuell übertragen. Als nichtmedikamentöse Behandlung wurden Psychotherapie, Physiotherapie, Ergotherapie und intermittierende Selbstkatheterisierung bei Inkontinenz gewertet. Spezifische Therapieverfahren bei Stuhlinkontinenz wurden nicht erfasst. Hinsichtlich der immunologischen Therapien sei auf eine Vorpublikation verwiesen [19].

Die Daten wurden zunächst in eine PostgreSQL-Datenbank (objekt-relationales Open Source Datenbanksystem) überführt $[20,21]$. Die statistische Auswertung erfolgte unter Verwendung von Python 3 mittels der freizugänglichen wissenschaftlichen Bibliotheken psycopg 2 (Zugriff auf PostgreSQL), Pandas (statistische Methoden) und openpyxl (Generierung von ExcelDateien).

\section{Ergebnisse}

Die Anzahl der jährlich dokumentierten Visiten betrug 201016 647; 201730834 und 201828917 Visiten. Die durchschnittliche Visitenzahl betrug 3,47 $\pm 2,44$ pro Jahr, der durchschnittliche Bobachtungszeitraum war im Median 5,07 \pm 4,46 Jahre. Die Basisdaten der Stichprobe von 21407 Patienten, differenziert nach den MS-Subtypen, sind in $>$ Tab. 1 dargestellt. Das Durchschnittsalter der Patienten betrug 49,0 \pm 13,0 Jahre, die Krankheitsdauer im Median 14,3 Jahre. Der durchschnittliche EDSS-Score der Gesamtstichprobe lag bei 2,8 (Range 0,5-8,0). In allen Subtypen waren Frauen mit 59-74\% überrepräsentiert.

Was die Symptomhäufigkeit betrifft, war die Fatigue in allen Subtypen das häufigste Symptom (RRMS 96\%; CIS 93\%; SPMS $93 \%$; PPMS 95\%, alle Patienten 96\%, s. \Abb. 1 und > Tab. 2), gefolgt von der Spastik bei der SPMS und PPMS. Weitere häufige Symptome waren Miktionsstörungen, Ataxie/Tremor, Schmerzen, kognitive Störung, Depression und Feinmotorikstörung.

Die Ergebnisse der Anteile der unbehandelten, medikamentös, nichtmedikamentös oder kombiniert behandelten Patienten mit den häufigsten Symptomen sind in \Tab. 3 aufgeführt.

Tab. 1 Basisdaten des Registers, aufgeteilt nach MS-Subtyp.

\begin{tabular}{|l|l|l|l|l|}
\hline & $\begin{array}{l}\text { Durchschnittsalter } \\
\text { (Mittelwert } \pm \text { SD) }\end{array}$ & Frauenanteil (\%) & $\begin{array}{l}\text { Krankheitsdauer in Jahren } \\
\text { (Mittelwert) }\end{array}$ & EDSS (Median) \\
\hline $\begin{array}{l}\text { Gesamt } n=21407 \\
(100 \%)\end{array}$ & $49,0( \pm 13,0)$ & 72,3 & $14,3( \pm 8,9)$ & 2,8 \\
\hline PPMS $n=1166(5,4 \%)$ & $62,6( \pm 11,6)$ & 59,0 & $17,2( \pm 10,1)$ & 5,2 \\
\hline RRMS $n=16425(76,7 \%)$ & $46,3( \pm 11,7)$ & 73,9 & $12,8( \pm 7,6)$ & 2,1 \\
\hline SPMS $n=3124(14,6 \%)$ & $60,2( \pm 10,8)$ & 68,9 & $22,7( \pm 9,9)$ & 5,7 \\
\hline CIS $n=692(3,2 \%)$ & $42,2( \pm 11,6)$ & 73,1 & $7,8( \pm 5,2)$ & 1,2 \\
\hline
\end{tabular}

-Tab. 2 CIS: Klinisch isoliertes Syndrom; RRMS: schubförmig remittierende MS; SPMS: Sekundär progrediente MS; PPMS: Primär progrediente MS.

\begin{tabular}{|c|c|c|c|c|c|}
\hline Symptomhäufigkeit in \% & Alle & RRMS & CIS & SPMS & PPMS \\
\hline Fatigue & 96 & 96 & 93 & 93 & 95 \\
\hline Spastik & 31 & 28 & 11 & 47 & 36 \\
\hline Miktionsstörungen & 16 & 12 & 4 & 34 & 35 \\
\hline Ataxie / Tremor & 16 & 12 & 2 & 34 & 31 \\
\hline Schmerzen & 15 & 13 & 7 & 31 & 25 \\
\hline Kognitive Störung & 13 & 10 & 2 & 26 & 21 \\
\hline Depression & 16 & 15 & 12 & 23 & 15 \\
\hline Okulomotorikstörung & 7 & 7 & 8 & 8 & 5 \\
\hline Defaekationsstörung & 3 & 2 & 1 & 7 & 5 \\
\hline Sex. Funktionsstörung & 1 & 2 & 0 & 7 & 4 \\
\hline Dysarthrie / Dysphonie & 2 & 1 & 0 & 5 & 8 \\
\hline Dysphagie & 1 & 1 & 0 & 1 & 3 \\
\hline Feinmotorikstörung & 4 & 5 & 0 & 20 & 13 \\
\hline
\end{tabular}




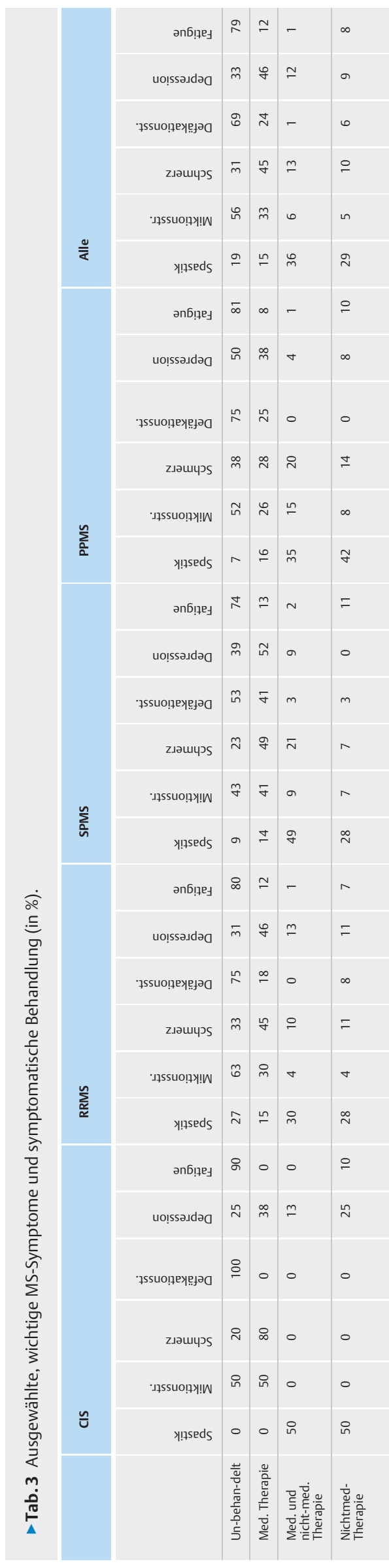

\section{Diskussion}

In den letzten 10 Jahren ist aus verschiedenen Motiven für Europa und Deutschland ein vermehrtes Interesse an der Gründung von „Real-world“-Krankheitsregistern im Allgemeinen und der MS im Speziellen festzuhalten. Die dargestellten Ergebnisse unserer NTD-Stichprobe zeigen dabei, dass mit einer auf die Herausforderung der Praxis zugeschnittenen Organisations- bzw. Infrastruktur und einer konsequenten Nutzung digitaler Datenerfassung eine valide Erhebung der wichtigsten Kerndaten der ambulanten Versorgung einer großen Stichprobe von MS-Patienten möglich ist. Insbesondere eine konsequente Umsetzung auf ein webbasiertes System mit einfacher Bedienbarkeit, professioneller IT-Betreuung und Datenschutz erlaubten eine über die Jahre konsequente und qualitätsgeprüfte Erhebung der Daten mit hoher Dichte und Validität unter den Arbeitsbedingungen der kassenärztlichfachärztlichen Routineversorgung.

Nach Gründung der ersten MS-Register in Europa in Dänemark 1996, Schweden 1997 und Norwegen 1998 publizierten für Deutschland Flachenecker et al. 2008 erste Registerdaten aus 57 Zentren (70\% Kliniken) und ca. 5500 Patienten [22]. Seither wurden die Datenbanken weiter ausgebaut. 2014 konnten hinsichtlich der MS in Europa 13, dann 2019 schon etwa 20 Register gezählt werden [23], [24]. Seither wurden die Datensammlungen weiterentwickelt. Aktuell gibt es in Deutschland allein etwa 6 neuroimmunologische Register [25].

Den Anfang bildete in Deutschland das schon 2001 gegründete Register des DMSG Bundesverbandes e.V. Seit einigen Jahren wird die Forschungsdatenbank durch die DMS-Stiftung des Bundesverbandes gepflegt und weiterentwickelt; diese hat aktuell fast 200 Zentren. Das Register erhebt die grundlegenden Daten der Stichprobe und führt Qualitäts- und Konsistenzkontrollen durch. Ein direkter Vergleich ist jedoch problematisch, da die Datenquellen und vermutlich auch die Datenerhebungen zu unterschiedlich sind. Die noch am ehesten vergleichbaren Parameter sind der Frauenanteil mit $72 \%$, das Überwiegen der RRMS-Verlaufsform und die Tatsache, dass die Fatigue immer das häufigste Symptom darstellt [14]. Ansonsten ist das Durchschnittsalter in beiden Stichproben unter 50 Jahre, die durchschnittliche MS-Dauer beträgt 13-14 Jahre, und der mediane EDSS war jeweils um 3. Somit erschien uns ein direkter statistischer Vergleich nicht sinnvoll, wäre aber für die Zukunft wünschenswert. Im internationalen Vergleich mit MSBase zeigt sich dann, dass in diesem Register die Patienten jünger und etwas geringer betroffen sind, zumindest legt dies der niedrigere EDSS-Wert nahe. Ein weiterführender Vergleich der Register ist in $>$ Tab. 4 dargestellt.

Weitere Gemeinsamkeiten beider deutscher Stichproben sind eine hohe Patientenzahl, eine hohe Anzahl von Konsultationen bzw. Visiten, Qualitätskontrollen, konsequente Nutzung einer webbasierten Datenbank, Queries und die Möglichkeit von Längsschnittuntersuchungen über mehrere Jahre. Für die Zukunft wäre es wünschenswert, angesichts der geografischen Variation ggf. ein Nord-Süd-Gefälle zu untersuchen; auch wäre ein Datenvergleich zwischen Ost- und Westdeutschland und ggf. eine Subgruppenanalyse der städtischen vs. ländlichen Versorgung von Interesse. 
Tab. 4 Registervergleich: DMSG, NTD und MSBase

\begin{tabular}{|c|c|c|c|}
\hline & $\begin{array}{l}\text { DMSG [14, } \\
26]\end{array}$ & NTD & $\begin{array}{l}\text { MSBase [27, } \\
\text { 28] }\end{array}$ \\
\hline $\begin{array}{l}\text { Anzahl } \\
\text { Patienten }\end{array}$ & $\begin{array}{l}22848-35 \\
755\end{array}$ & 21407 & 35869 \\
\hline $\begin{array}{l}\text { Durch- } \\
\text { schnittsalter }\end{array}$ & $46,3 \pm 12,2$ & $49,0 \pm 13,0$ & $43 \pm 12,0$ \\
\hline Frauenanteil & $72 \%$ & $72 \%$ & $71 \%$ \\
\hline $\begin{array}{l}\text { Krankheits- } \\
\text { dauer in } \\
\text { Jahren }\end{array}$ & $12,8 \pm 9,8$ & $14,3 \pm 8,9$ & $9,9(4,6-17)$ \\
\hline $\begin{array}{l}\text { Medianer } \\
\text { EDSS (Range) }\end{array}$ & 3,0 & $2,8(0,5-8)$ & $2,5(1,5-5)$ \\
\hline PPMS & $6,4 \%-6,5 \%$ & $5,4 \%$ & $5,0 \%$ \\
\hline RRMS & $\begin{array}{l}59,5 \%-73,3 \\
\%\end{array}$ & $76,7 \%$ & $72,0 \%$ \\
\hline SPMS & $16,0 \%-21,8 \%$ & $14,6 \%$ & $11,8 \%$ \\
\hline CIS & $2,0 \%-4 \%$ & $3, \%$ & $9,4 \%$ \\
\hline $\begin{array}{l}\text { Stichprobe / } \\
\text { Land }\end{array}$ & Deutschland & Deutschland & International \\
\hline Ärzte & $>186 \#$ & 133 & 609 \\
\hline $\begin{array}{l}\text { Kliniken, Zen- } \\
\text { tren, Praxen }\end{array}$ & 186 & 66 & 139 \\
\hline
\end{tabular}

Legende: \#Genaue Daten nicht sinnvoll zu erheben, da die Anzahl der Ärzte, z. B. in einer MS-Ambulanz, einer Fluktuation unterworfen ist (pers. Mitteilung DMSG 12.12.19).

Neben der Fatigue (58\%) als häufigstes Symptome der letzten dokumentierten Untersuchung des DMSG-Registers waren Spastik (48\%), Miktionsstörungen (44\%), Ataxie/Tremor (37\%), Schmerzen (34\%), kognitive Störungen (33\%) und Depressionen (32\%) häufig [14]. Hinsichtlich Symptomhäufigkeit ist in der hiesigen Stichprobe die Fatigue (96\%) noch häufiger, während Spastik (31\%), Schmerzen (15\%), Miktionsstörungen (16\%), Ataxie und Tremor (16\%) hier allesamt seltener waren. Die Unterschiede sind nicht unerheblich, aber nach unserer Meinung erklärbar durch die unterschiedlichen Datenquellen, die zwar meist standardisierten, im Einzelfall aber vermutlich doch unterschiedlichen Erhebungsmethoden, ggf. im Einzelfall unterschiedliche EDSSRanges, ggf. unterschiedliche Erhebungsweise der Fatigue oder durch häufiger durchgeführte urologische Funktionsdiagnostik in den Kliniken vs. Praxis. Sie stellen aber sicher eine Limitation unserer Ergebnisse dar.

Weitere Unterschiede betreffen die Behandlungsdaten der symptomatischen Therapie. So zeigen unsere Daten, dass bei der RRMS, der häufigsten Verlaufsform, ein recht gut operationalisierbares Symptom „wie die Spastik“ in 27 \% unbehandelt bleibt. Für die ganze Population zeigte sich ein Wert von $19 \%$. Bei genauerer Betrachtung der Daten der DMSG zeigen sich dort auch Werte über $20 \%$ [14]. Dies lässt sich ggf. durch einen Teil von Patienten erklären, bei denen sich zwar in der Untersuchung eine Spastik zeigen ließ, die sich aber dadurch noch durch ggf. Schmerzen in ihrer Lebensqualität eingeschränkt fühlen. Noch erheblicher war der Befund, dass bei $63 \%$ der RRMS-Patienten und bei $56 \%$ der Gesamtpopulation eine Blasenstörung nicht behandelt wurde. In der DMSG-Stichprobe lag dieser Wert bei ca. 44\% [14]. Auch hier ist das unterschiedliche Setting, ein geringerer Bedarf oder seltenere Nachfragen nach entsprechender Therapie in den Praxen, Möglichkeit der Therapieübernahme (z. B. für Physiotherapie) durch Hausärzte, andererseits die abweichenden diagnostischen und therapeutischen Möglichkeiten der multiprofessionellen Teams in den Kliniken (Physiotherapie, Ergotherapie) die wahrscheinlichste Erklärung der Unterschiede. Letztlich sind Lücken in der ambulanten Versorgung nicht auszuschließen.

Ein weiteres wichtiges Ergebnis betraf die Häufigkeit der kognitiven Störungen, die immerhin in $26 \%$ der Patienten mit SPMS und in $13 \%$ der Gesamtstichprobe auftrat. Die Häufigkeit war in der DMSG-Stichprobe sogar noch höher (ca. 33 \% [14]). In unserer Stichprobe liegen dazu zu wenige Behandlungsdaten vor. In der DMSG-Stichprobe blieben über $70 \%$ unbehandelt, unsere Daten dürften aber ähnlich sein oder noch darüber. Hier sollte vermehrt über eine ambulante neuropsychologische Therapie oder Ergotherapie für dieses wichtige Symptom nachgedacht werden.

Mit qualifizierten Versorgungsforschungsdaten können klinische Fragestellungen nach Neuzulassung von Medikamenten sowie Wirksamkeitsvergleiche zwischen Therapien mit validierten Methoden auf dem Niveau klinischer Studien bzw. nach den Prinzipien der guten pharmakoepidemiologischen Praxis bearbeitet werden. Durch Einbeziehung von großen Versorgungsforschungsdatenbanken könnte es gelingen, neue Instrumente zur Realisierung einer mehr personalisierten Medizin zu entwickeln, wie die letzten Projekte von NTD wie PHREND und DESTINY zeigten. Auf Grundlage hoher Standards, vor allem im Datenschutz, wie durch die EU-DSGVO vorgegeben und die EMA-Patient-Registries-Initiative verlangt, können neue Entwicklungen hin zu einer Harmonisierung der Datenerfassung und, darauf basierend, einer europaweiten Integration von Versorgungsdaten aus unterschiedlichen Ländern in regulatorische Verfahren von und nach Zulassung neuer Substanzen angestoßen werden.

Wünschenswert wäre ein noch tieferer Vergleich unseres Registers mit internationalen Ergebnissen, wie dem MSBase-Register. Ein Datenabgleich der verschiedenen Register, zumindest eine Harmonisierung, ggf. gemeinsame Projekte wären geeignete Mittel, um einen noch umfassenderen Einblick in den gegenwärtigen Stand der Versorgung der MS-Population zu erhalten.

\section{Fazit für die Praxis}

- Das NeuroTransData Netzwerk (NTD) niedergelassener Neurologen und Nervenärzte hat seit 2008 deutschlandweit demografische und klinische Informationen von über 21000 MSPatienten mit hoher Datendichte und Validität erfasst.

- Fatigue ist das häufigste Symptom in beiden Stichproben.

- Die Mehrzahl der ambulanten Patienten erhält eine oder verschiedene Formen der Therapie für häufige Symptome wie Spastik (81\% behandelt), Schmerz (69\% behandelt) und Depression (67\% behandelt). 
- Miktionsstörungen sind vor allem bei der SPMS und PPMS häufig (34\% bzw. $35 \%$ ), bleiben aber in über $50 \%$ unbehandelt.

- Defäkationsstörungen sind seltener (bis $7 \%$ bei SPMS), bleiben aber auch oft (bis $75 \%$ ) unbehandelt.

- Diese Versorgungsdaten identifizieren spezifischen Handlungsbedarf (unmet needs) hinsichtlich der pharmakologischen und nichtpharmakologischen symptomatischen MS-Therapie in der ambulanten Behandlung.

\section{Interessenkonflikt}

Die Autoren geben an, dass keine Interessenkonflikte vorliegen.

\section{Literatur}

[1] Montalban X, Gold R, Thompson AJ et al. ECTRIMS / EAN guideline on the pharmacological treatment of people with multiple sclerosis. Eur ] Neurol 2018; 25: 215-237

[2] Flachenecker P1, Kobelt G2, Berg J3 et al. European Multiple Sclerosis Platform. New insights into the burden and costs of multiple sclerosis in Europe: Results for Germany. Mult Scler 2017; 23 (suppl. 2): 78-90

[3] Rotstein DL, Chen H, Wilton AS et al. Temporal trends in multiple sclerosis prevalence and incidence in a large population. Neurology 2018 90 16: e1435-e1441

[4] GBD 2016 Multiple Sclerosis Collaborators. Global, regional, and national burden of multiple sclerosis 1990-2016: a systematic analysis for the Global Burden of Disease Study 2016. Lancet Neurol. 2019; 18 (3): 269-285

[5] Petersen G1, Wittmann R, Arndt V et al. Epidemiology of multiple sclerosis in Germany: regional differences and drug prescription in the claims data of the statutory health insurance. Nervenarzt 2014; 85 (8): 990-998

[6] Holstiege J, Steffen A, Goffrier B et al. Epidemiologie der Multiplen Sklerose - eine populationsbasierte deutschlandweite Studie. 2017. https://www.versorgungsatlas.de/fileadmin/ziva_docs/86/VA-86-M ultiple\%20Sklerose-Bericht-V12_1.pdf; Stand: 24.10.2018

[7] https://www.g-ba.de/institution/presse/pressemitteilungen/210/; Stand: 18.08 .2018

[8] https://www.bundesgesundheitsministerium.de/service/begriffe-von-a-z/v /versorgungsstrukturgesetz.html; Stand: 18.08.2018

[9] https://www.iges.com/presse/2016/weissbuch-ms/index_ger.html; Stand: 20.02 .2019
[10] Koch-Hendriksen N. The Danish Multiple Sclerosis Registry: a 50-year follow up. Multiple Sclerosis 1999; 5: 293-296

[11] Flachenecker P, Stuke K, Elias $W$ et al. Multiple-Sklerose-Register in Deutschland Ausweitung des Projektes 2005/2006. Dtsch Arztebl 2008; 105 (7): 113-119

[12] Zettl U, Eichstädt K, Ellenberger D et al. MS in Deutschland: Symtome und Behandungsdefizite. NeuroTransmitter 2018; 29 (6): 42-44

[13] https://www.ema.europa.eu/en/human-regulatory/post-authorisation/ patient-registries; registries; Stand: 27.02.2019

[14] https://www.ema.europa.eu/documents/report/report-multiple-sclero sis-registries_en.pdf; Stand: 27.02.2019

[15] https://www.msbase.org/; Stand: 19.09.2019

[16] https://www.neurotransdata.com/ueber-uns; Stand: 12.12 .2019

[17] https://www.neurotransdata.com/ueber-uns; Stand: 09.09.2019

[18] Penner IK, Raselli C, Stöcklin M et al. The FSMC (Fatigue Scale for Motor and Cognitive Functions) - validation of a new instrument to assess MS related fatigue in clinical routine. Multiple Sclerosis 2009; 15: 15091517

[19] Braune S1, Grimm S2, van Hövell P2 et al. NTD Study Group Comparative effectiveness of delayed-release dimethyl fumarate versus interferon, glatiramer acetate, teriflunomide, or fingolimod: results from the German NeuroTransData registry. J Neurol 2018; 265 (12): 2980-2992

[20] https://de.wikipedia.org/wiki/Representational_State_Transfer; Stand: 20.02.2019

[21] https://www.postgresql.org; Stand: 20.02.2019

[22] Flachenecker P, Stuke K, Elias W et al. Multiple-Sklerose-Register in Deutschland Ausweitung des Projektes 2005/2006. Dtsch Arztebl 2008; 105 (7): 113-119

[23] Bezzini D, Battaglia MA. Multiple Sclerosis Epidemiology in Europe. Adv Exp Med Biol 2017; 958: 141-159. doi: 10.1007/978-3-319-47861-6_9

[24] Glaser A, Stahmann A, Meissner T et al. Multiple sclerosis registries in Europe - An updated mapping survey. Mult Scler Relat Disord 2019; 27 : $171-178$

[25] Thiel S, Leypoldt F, Röpke L et al. Neuroimmunological Registries in Germany. Neurol Int Open 2018; 2: E25-E39

[26] https://www.msregister.de/fileadmin/user_upload/MS-Registerueber sicht_2019.pdf; Stand: 12.12.2019

[27] Kalincik T, Manouchehrinia A, Sobisek L et al. MSBase Study Group. Towards personalized therapy for multiple sclerosis: prediction of individual treatment response. Brain 2017; 140 (9): 2426-2443. doi:10.1093/brain/awx185

[28] Data quality evaluation for observational multiple sclerosis registries. Kalincik T, Kuhle J, Pucci E et al. MSBase Scientific Leadership Group and MSBase Study Group. Mult Scler 2017; 23 (5): 647-655 